\title{
A case report of an immunocompetent patient with Coccidioides meningoencephalitis with atypical brain magnetic resonance imaging findings during a 1 year follow-up
}

\author{
Dongkwan Jin MD, Juliana Gomez MD
}

\begin{abstract}
Coccidioides meningoencephalitis is a central nervous system (CNS) fungal infection with Coccidioides species which can lead to various CNS complications, such as hydrocephalus, vasculitis, and stroke. Most cases reported with Coccidioides meningoencephalitis were in immunocompromised patients, and the radiologic characteristics on this condition are not well established. Here we report a case of Coccidioides meningoencephalitis in an immunocompetent patient with one-year follow-up brain magnetic resonance image (MRI) studies after successful treatment with antifungal drugs. The MRI demonstrated subcortical parenchymal lesions, selectively involving the white matter, and persistent meningeal enhancement.
\end{abstract}

Keywords: Coccidioides, Coccidioidomycosis, meningoencephalitis, immunocompetent, MRI

\section{INTRODUCTION}

Coccidioidomycosis (Valley Fever, San Joaquin Valley Fever, desert rheumatism, or Posadas-Wernicke Disease) is a fungal infection caused by Coccidioides immitis or Coccidioides posadasii. Coccidioides is a dimorphic, soil-borne, ascomycete fungus that grows in dry regions with sandy, alkaline soils. Endemic areas include California, Arizona, New Mexico, Texas, Central America, and South America. C. immitis occurs more frequently in California; C. posadasii is common in Texas, Central America, and South America. ${ }^{1}$

While exposure to Coccidioides species is prevalent in endemic areas, symptomatic disease occurs in only one third of exposed individuals, typically presenting as a localized pulmonary process. Disseminated

Corresponding author: Dongkwan Jin

Contact Information: Doctor.jin@ttuhsc.edu DOI: $10.12746 /$ swrccc.v7i28.542 disease, including coccidioidal meningitis, occurs in less than $5 \%$ of symptomatic individuals. ${ }^{2}$ Central nervous system involvement is reported in approximately one third to one half of patients with disseminated disease, even in immunocompetent individuals, and it is one of the most devastating complications of coccidioidomycosis, associated with significant morbidity and, sometimes, mortality. ${ }^{3}$ We are reporting patient who developed Coccidioides meningitis with significant cerebral involvement who had abnormal magnetic resonance imaging studies 1 year following the initiation of his treatment.

\section{CASE}

A 24-year-old, previously healthy, Hispanic man presented with headache for one month. The patient was from New Mexico, where he worked as a pipefitter. Initial brain magnetic resonance images (MRI) with and without contrast showed mild dilation of the lateral ventricles, minimal periventricular edema, and diffuse leptomeningeal enhancement; these findings were consistent with hydrocephalus and meningitis. 

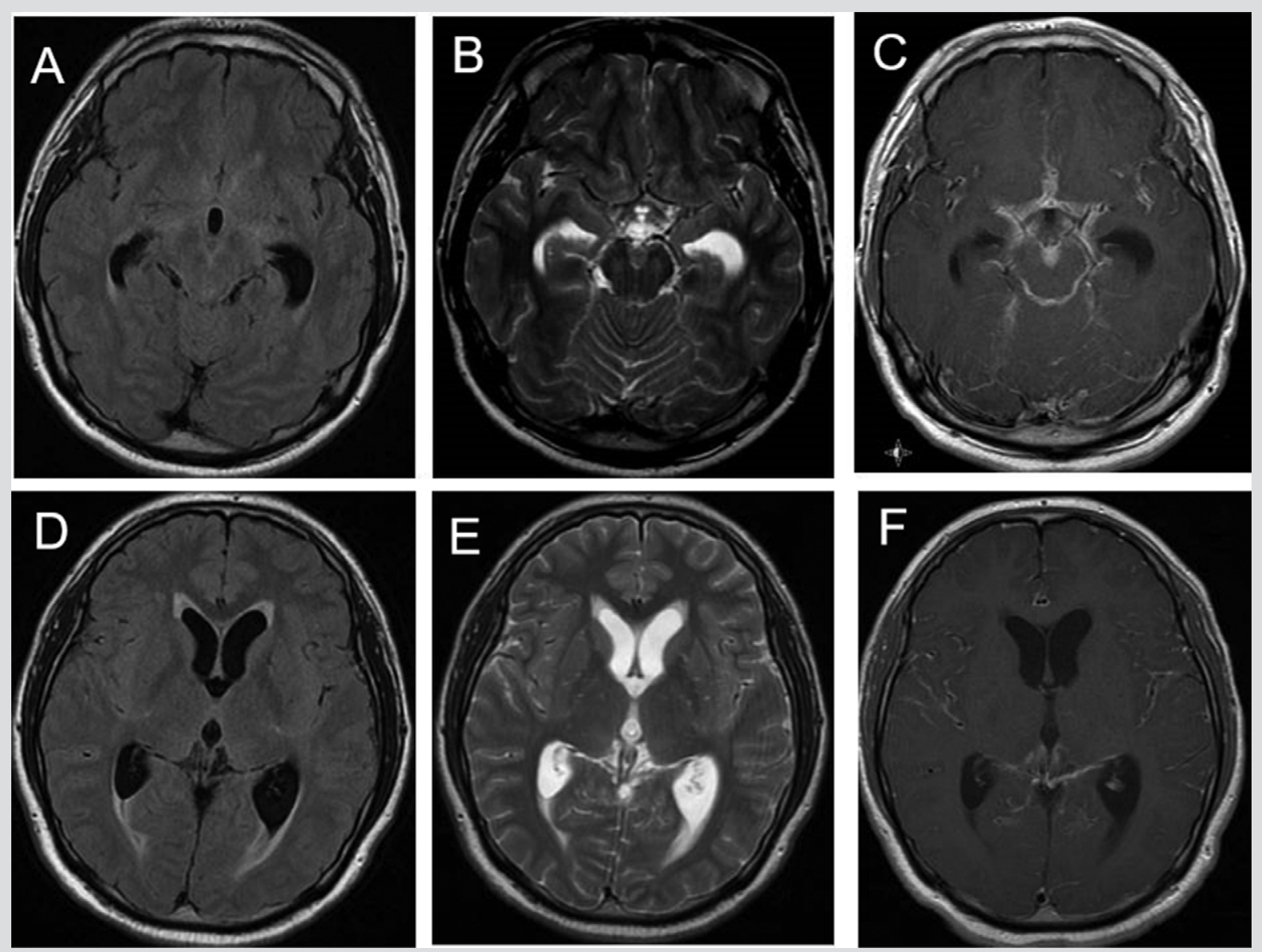

Figure 1. Initial brain MRI study. FLAIR (A, D) and T2 (B, E) axial plane MRI images showing mild dilation of the lateral ventricles along with minimal periventricular edema. No parenchymal lesions were found at this point. Contrast enhanced T1 (C, F) axial plane MRI images showed diffuse, abnormal leptomeningeal enhancement, consistent with meningitis.

Lumbar puncture and cerebral spinal fluid (CSF) studies showed a high opening pressure of $40 \mathrm{~cm} \mathrm{H}_{2} \mathrm{O}$ (normal range: 5-20 $\mathrm{cm} \mathrm{H}_{2} \mathrm{O}$ ), $\mathrm{RBC} 19 / \mathrm{mm}^{3}$ (normal range: $<5 / \mathrm{mm}^{3}$ ), WBC $160 / \mathrm{mm}^{3}$ (normal range: $<5 / \mathrm{mm}^{3}$ ) with $93 \%$ lymphocytes, proteins $68 \mathrm{mg} / \mathrm{dL}$ (normal range: 15-45 mg/dL), and glucose $24 \mathrm{mg} / \mathrm{dL}$ (normal range: $50-80 \mathrm{mg} / \mathrm{dL}$ ) with a serum glucose of $119 \mathrm{mg} / \mathrm{dl}$. Coccidioides antibodies (by complement fixation and immunodiffusion) were positive in both the CSF and serum.

Extensive studies were conducted for evaluation of disseminated Coccidioidomycosis, including a skin biopsy on a verrucous nodule on his left third finger which revealed cutaneous Coccidioidomycosis. A computed tomography scan of the chest showed prevascular and perihilar lymphadenopathy but no pulmonary infiltrates. Culture from bronchial washing was positive for Coccidioides species. Initial brain MRI study showed signs of basal meningitis and hydrocephalus, but there was no evidence of parenchymal lesions (Figure 1). A magnetic resonance angiography study of head did not find evidence of vasculitis.

He was treated with fluconazole initially. However, it had to be discontinued due to an increase in liver 

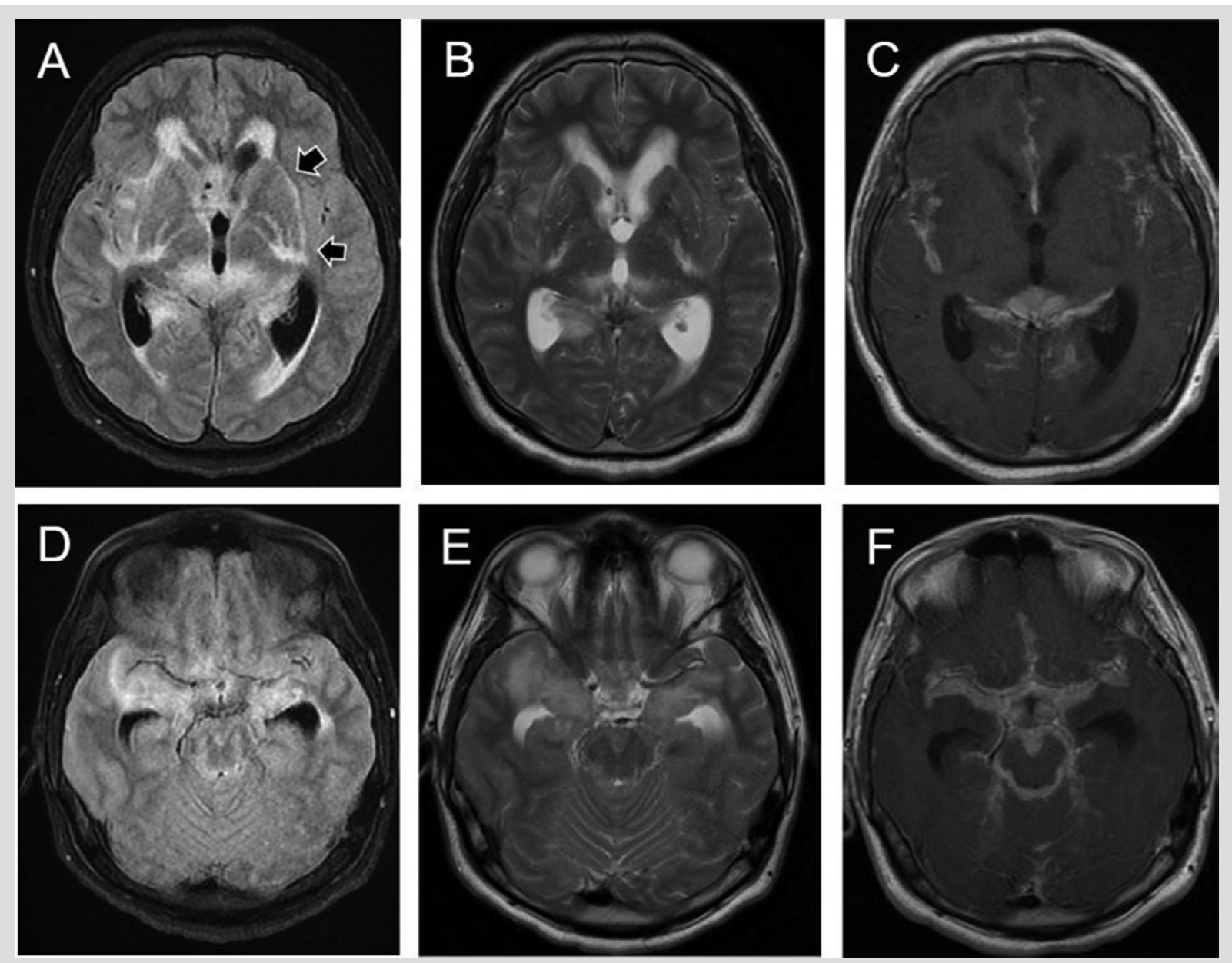

Figure 2. Brain MRI after 6 weeks of antifungal treatment. FLAIR (A, D) and T2 (B, E) images showed extensive lesions of high signal intensity around bilateral thalami cerebral peduncles and mesial temporal lobes. Of note, white matter lesion is extending to internal and external capsules as well as periventricular white matter, sparing adjacent subcortical grey matters (arrows). Postcontrast $(\mathrm{C}, \mathrm{F})$ images showed persistent leptomeningeal enhancement.

enzymes and the lack of significant clinical improvement. He was then treated with intravenous and intrathecal amphotericin B for approximately two months. Voriconazole was added two months after his initial presentation and has been continued for over one year.

His clinical course was complicated with 2 episodes of status epilepticus, a decline in cognitive function, psychosis, incoordination, and tremors. A follow-up brain MRI six weeks after the initiation of antifungal agents showed extensive white matter changes involving the basal ganglia, mesial temporal cortices, thalami, cerebral peduncles, and pons (Figure 2).

The one-year follow-up brain MRI continued to show dense enhancement of meninges (Figure 3). A repeat CSF study one year after onset of symptoms showed a mild elevation of protein $(99 \mathrm{mg} / \mathrm{dL}$; normal range: $15-45 \mathrm{mg} / \mathrm{dL}$ ) without pleocytosis. He showed remarkable improvement after several months of treatment but had some residual symptoms, including a slight decrease in fine motor skills and mild short-term 

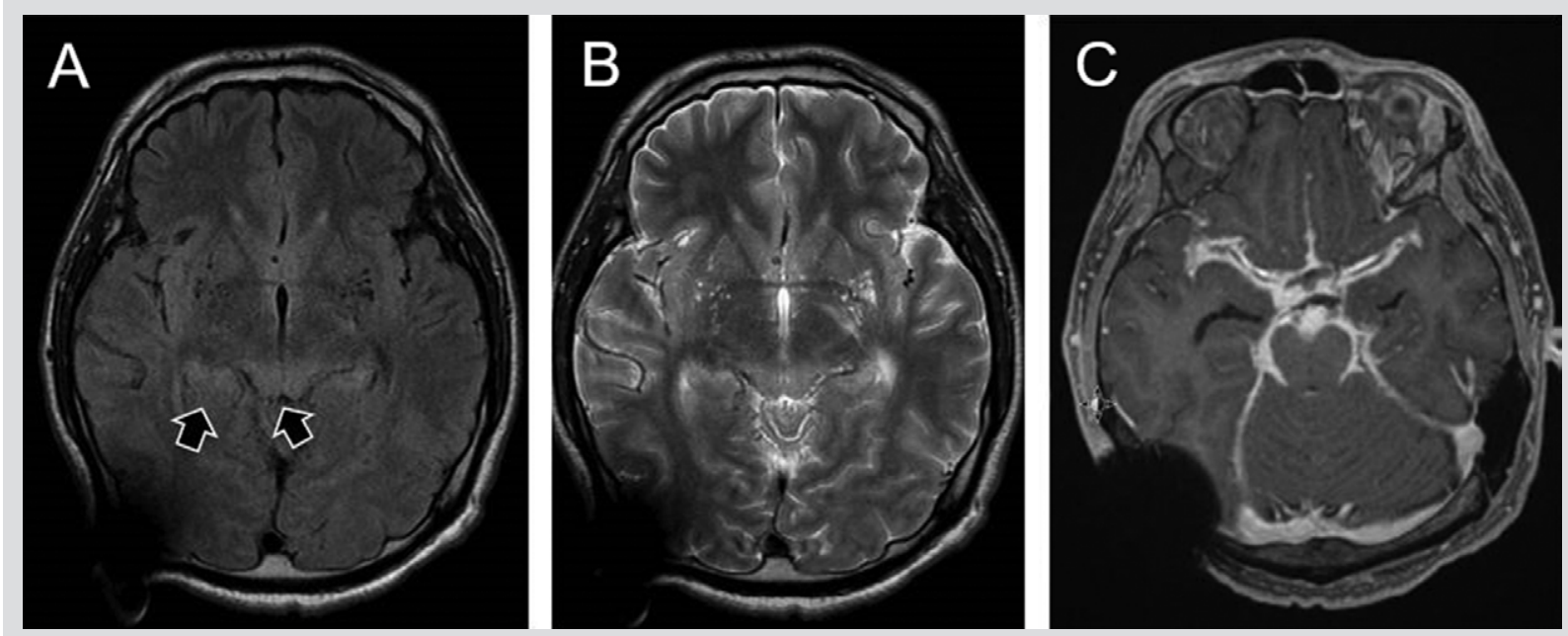

Figure 3. Follow-up images 1 year after initial presentation. On FLAIR (A) and T2 (B), most of the parenchymal lesions had disappeared, except the lesions in the posterior half of the midbrain and bilateral entorhinal cortices. Hyperintense lesions in the basal ganglial white matter and temporal lobes resolved but the high signal intensity in the cerebral peduncles remained (arrows). The leptomeningeal enhancement persisted (C) despite clinical and serological improvement. There is no evidence of hydrocephalus. The pulse artifact at the right parietal lobe is due to ventriculoperitoneal shunt.

memory problems. He was completely independent in his activities of daily living.

\section{Discussion}

Up to $70 \%$ of the population in endemic areas has been infected by Coccidioides species, but most infections are mild or asymptomatic. The lungs are the most common organs involved in isolated Coccidioides infection. However, our case demonstrates Coccidioides should be considered as a primary cause of meningitis if a person resides in an endemic area, even if the individual is young and immunocompetent and has no signs of respiratory involvement. It is especially important for physicians in these areas to recognize the clinical and radiological manifestations of this potentially fatal condition in a timely manner.

Central nervous system involvement presents as meningitis or meningoencephalitis. Headache, fever, nausea, vomiting, and meningeal irritation signs can be subacute or chronic, developing over several weeks, like in this case. The condition can be complicated by hydrocephalus (in $30-50 \%$ of patients), vasculitis and cerebral infarction (in 15-20\% of patients), abscess, intracranial aneurysm, subarachnoid hemorrhage, and venous thrombosis.,

Coccidioidal meningitis, defined according to the National Institute of Allergy and Infectious DiseasesMycoses Study Group, includes the following criteria: (1) isolation of $C$. immitis from the CSF; (2) complement fixing anti-coccidioidal antibodies detected in the CSF in the presence of other CSF abnormalities typical of coccidioidal meningitis; or (3) illness plus CSF abnormalities compatible with chronic meningitis and either detection of serum complement fixing type antibodies or isolation of $C$. immitis from an extraneural site. Aggressive treatment with an antifungal drug is essential. Nevertheless, the prognosis is often poor, since CNS complications, especially hydrocephalus, frequently occur despite antifungal treatment. Moreover, relapses are very common, even years after treatment, and life-long treatment is required in most cases. ${ }^{5}$

The images of this patient demonstrate MRI findings of severe Coccidioides meningoencephalitis, 
including prominent leptomeningeal enhancement, mainly on the basal cistern, dilatation of ventricles, transependymal edema (also called interstitial cerebral edema), and hydrocephalus. Our case has two unique and atypical features for Coccidioides meningoencephalitis on his brain MRI: 1) Diffuse, selective white matter lesions without extension into the subcortical grey matter. The patient had extensive parenchymal brain lesions, evident in FLAIR and T2-weighted imaging, involving the internal and external capsule, paraventricular white matter, mesial temporal lobe, and midbrain. The distribution of these parenchymal lesions was symmetric, and there was no corresponding diffusion restriction. Previous studies of multiple patients with Coccidioides infection with CNS involvement have reported midbrain or pontine involvement. But, this is the first case report with high signal intensity in the internal and external capsule of basal ganglia, without involvement of subcortical grey matters (lentiform nuclei and caudate nuclei). The appearance of selective damage through the internal and external capsule, as well as the paraventricular white matter, is similar to the radiologic findings of leukodystrophic conditions, and the absence of diffusion restriction in this area argues against an ischemic or vasculitic nature of the lesions. 2) Persistent pachymeningeal enhancement after one year of treatment with clinical and serological improvement. It was very interesting that despite clinical, serological, and CSF test improvement, the brain MRI continued to show dense meningeal enhancement. We could not find any previous reports on persistent dural enhancement after several months of treatment with good recovery. It is perhaps too early to find complete resolution of meningeal enhancement. However, prolonged meningeal enhancement, such as in this case, does not necessarily have a clinical correlation, and this is important to keep in mind to avoid considering these cases as treatment failures and to avoid giving erroneous predictions in disease prognosis.
The current case showed a variety of radiologic findings in acute, subacute and chronic stages of Coccidioides meningoencephalitis with successful treatment response. This is the first report on long-term follow-up imaging studies for Coccidioides meningoencephalitis to our best knowledge. Studies, such as histopathologic correlation and clinical impact in association with radiologic finding, are needed.

Article citation: Jin D, Gomez J. A case report of an immunocompetent patient with Coccidioides meningoencephalitis with atypical brain magnetic resonance imaging findings during a 1 year follow-up. The Southwest Respiratory and Critical Care Chronicles 2019:7(28):33-37

From: The Department of Neurology at Texas Tech University Health Sciences Center, Lubbock, Texas

Submitted: $7 / 22 / 2018$

Accepted: 4/3/2019

Reviewers: Mark Lacy MD, Mirla Avila MD

Conflicts of interest: none

This work is licensed under a Creative Commons

Attribution-ShareAlike 4.0 International License.

\section{REFERENCES}

1. Murthy JM, Sundaram C. Fungal infections of the central nervous system. Handb Clin Neurol 2014;121:1383-1401.

2. Galgiani JN, Ampel NM, Blair JE, et al. Coccidioidomycosis. Clin Infect Dis 2005;41(9):1217-1223.

3. Johnson RH, Einstein HE. Coccidioidal meningitis. Clin Infect Dis 2006;42(1):103-107.

4. Kleinschmidt-DeMasters BK, Mazowiecki M, et al. Coccidioidomycosis meningitis with massive dural and cerebral venous thrombosis and tissue arthroconidia. Arch Pathol Lab Med 2000;124(2):310-314.

5. Dewsnup DH, Galgiani JN, Graybill JR, et al. Is it ever safe to stop azole therapy for Coccidioides immitis meningitis? Ann Intern Med 1996;124(3):305-310. 